\title{
SAND99-1036C
}

RECEIVES

OCT 181999

USTI

\section{COMPUTATIONAL MODELING OF HETEROGENEOUS REACTIVE MATERIALS AT THE MESOSCALE*}

\author{
M. R. Baer \\ Energetic and Multiphase Processes, Dept. 9112 \\ Sandia National Laboratories, Albuquerque,NM, 87185
}

\begin{abstract}
The mesoscopic processes of consolidation, deformation and reaction of shocked porous energetic materials are studied using shock physics analysis of impact on a collection of discrete "crystals." Highly resolved three-dimensional CTH simulations indicate that rapid deformation occurs at material contact points causing large amplitude fluctuations of stress states with wavelengths of the order of several particle diameters. Localization of energy produces "hot-spots" due to shock focusing and plastic work near internal boundaries as material flows into interstitial regions. Numerical experiments indicate that "hot-spots" are strongly influenced by multiple crystal interactions. Chemical reaction processes also produce multiple wave structures associated with particle distribution effects. This study provides new insights into the micromechanical behavior of heterogeneous energetic materials strongly suggesting that initiation and sustained reaction of shocked heterogeneous materials involves states distinctly different from single jump state descriptions.
\end{abstract}

\section{INTRODUCTION}

Nearly all energetic materials, including explosives, pyrotechnics, propellants, and intermetallics are heterogeneous in nature and often consist of a mixture of poly-crystalline and binder materials. These energetic materials exhibit distinctly different thermal/mechanical/chemical behavior than pure materials because microstructure introduces internal boundary effects at the mesoscale. For high explosives, the shock sensitivity of initiation and sustained reaction is known to be controlled by processes occurring at the crystal level (1). For example, the threshold to reaction can be greatly influenced by changes in crystal morphology, defect content and size distribution $(2,3)$.

Time-resolved measurements (4) of the shock wave structure in heterogeneous materials have provided much insight in the high strain rate processes associated with impact on granular explosives; however, these measurements resolve averaged wave fields associated with the interac1

\footnotetext{
- Sandia is a multiprogram laboratory operated by Sandia Corporavion. a Lockheed Martin Company, for the U.S. Department of Ejergy under contract DE-ACO4 94AL85000.
}

tions of internal boundaries. At the crystal scale, the initiation of heterogeneous materials takes place because shocks interact with material heterogeneities, causing "hot-spots" that lead to reaction. Localization of energy produces associated spacetime fluctuations in the thermodynamic fields (such as pressure and temperature). When averaged over a sufficiently large space, these effects produce an energy release that is a combination of mechanics, dissipation and chemistry. A variety of physical phenomena result in the creation of "hot-spots" and it is generally agreed that plastic deformation is the key feature of the energy localization. This deformation is due to material rearrangement near boundaries or at internal dislocations within crystals. The prevailing theory of shock-induced "hotspots," is based on the notion of phonon energy transport/dissipation along moving dislocations (5). This atomic-level view, by itself, may be too simplistic; the chemical and physical processes associated with "hot-spots" involve space-time fluctuating states at the mesoscale whereby multiple crystals interact to produce multiple shocks significantly different than single jump states. 


\section{DISCLAIMER}

This report was prepared as an account of work sponsored by an agency of the United States Government. Neither the United States Government nor any agency thereof, nor any of their employees, make any warranty, express or implied, or assumes any legal liability or responsibility for the accuracy, completeness, or usefulness of any information, apparatus, product, or process disclosed, or represents that its use would not infringe privately owned rights. Reference herein to any specific commercial product, process, or service by trade name, trademark, manufacturer, or otherwise does not necessarily constitute or imply its endorsement, recommendation, or favoring by the United States Government or any agency thereof. The views and opinions of authors expressed herein do not necessarily state or reflect those of the United States Government or any agency thereof. 


\section{DISCLAIMER}

Portions of this document may be illegible in electronic image products. Images are produced from the best available original document. 
Much of the current work in computational modeling of heterogeneous material describes macroscale behavior based on ensemble-averaged continuum theory $(6,7)$. At the atomistic scale microscopic behavior has also been theoretically studied using molecular dynamics methods. Unfortunately, both descriptions fall short of completely describing the fundamental processes associated with the dispersive nature of reactive waves in heterogeneous media. The mesoscale has not been as extensively studied; yet, it is the level that bridges continuum and atomistic scales. Shock physics analysis can now take advantage of new parallel computing architecture to provide enhanced resolution of shock processes at the mesoscale.

This work presents three-dimensional numerical simulations of shock impact on a collection of poly-crystalline grains. Detailed wave fields are studied including the effects of material strength, thermal dissipation and reaction.

\section{MESOSCALE MODELING}

Three-dimensional shock wave structure in heterogeneous material is investigated using the Eulerian CTH shock physics code (8) for a mixture of "crystals" and binder having multi-material disordered geometry and several relevant aspects of material physics. Although most poly-crystalline materials are known to have structural regularity and directional-dependent material properties, this work considers materials with idealized morphology and isotropic properties.

In constructing a material configuration the statistical nature of the heterogeneous media is represented by a computational geometry describing an ensemble of "crystals" with varied size distribution consolidated to a random closely packed configuration. In real materials, microphotographs indicate that individual particles consist of a myriad of shapes varying from spherical, polyhedral, needleshaped or platelets. This work models the shock behavior for a collection of "crystals" with polyhedral geometry.
To generate a random packing of arbitrary polyhedral "crystals" several strategies have been considered. Initially a drop-and-fill method was used whereby an arbitrary number of spherical particles is introduced into a random space and the rigid body motion, under the influence of "gravity," leads to interbody collision and contact frictional effects (9). Unfortunately, this produces a stereological system with a directional bias and nonuniformities near boundaries; thus an alternative strategy was sought.

A more efficient means of generating a random ensemble of particles is based on the statistical mechanical theory of liquids $(10,11)$. In this approach, Monte Carlo (MC) and molecular dynamics (MD) methods are used to generate closely packed particles of arbitrary distributions. Briefly, a large region of three-dimensional space is filled by placing a specified number of particles on the lattice sites of a simple cubic array. The particle size is chosen randomly from a prescribed discrete distribution. The starting packing fraction is chosen to be sufficiently low such that the initial configuration is free of any particle overlap. The particles are then given random velocities and a hard sphere molecular dynamics simulation is conducted using a standard collision algorithm. Given the low packing fraction, the initial lattice configuration quickly disintegrates and evolves to a low density equilibrated fluid mixture. This equilibrium configuration forms the starting point for an efficient densification $M D$ run in which the density is increased dynamically until the particles are essentially locked in place. The densification algorithm employs growing particles whose size increases at a constant rate, while the particles continue to move through space and undergo collisions. The MD continues until the packing configuration reaches a specified target volume fraction or a maximum packing condition.

Class A HMX particles having an unpressed particle size distribution (12) for Holston lot 92032 is chosen as a representative energetic material. Closely-packed configurations are determined 
using the $\mathrm{MC} / \mathrm{MD}$ method considering five classes of particle sizes (fine particles are lumped into the smallest size category). In applying the packing algorithm, the ensemble of HMX "crystals" reaches a closely-packed limit density of approximately 65\% TMD. (To achieve higher densities, mechanical deformation/fragmentation of the "crystals" takes place or, alternatively, interstitial regions are filled with binder.)

As a simplification, isotropic properties are used to describe the crystalline HMX material. Each particle size category is assigned a separate set of equation of state and material strength descriptions to accommodate interactions of material boundaries. Material boundary interactions are treated by imposing mixing rules for the yield strength within computational cells containing material interfaces. A frictionless material interface is represented by imposing a zero material yield in mixed-material computational cells. Alternatively, a volume fraction-weighted yield strength constraint produces material interfaces that are continuously bonded.

Table 1. HMX EOS/Strength/Fracture Parameters

\begin{tabular}{ll}
\hline \multicolumn{1}{c}{ Parameter } & \multicolumn{1}{c}{ Value } \\
\hline Particle Size Distribution & Weight fraction: \\
$<44 \mu \mathrm{m}$ & 0.092 \\
$62 \mu \mathrm{m}$ & 0.121 \\
$88 \mu \mathrm{m}$ & 0.118 \\
$125 \mu \mathrm{m}$ & 0.278 \\
$177 \mu \mathrm{m}$ & 0.182 \\
$250 \mu \mathrm{m}$ & 0.156 \\
$>350 \mu \mathrm{m}$ & 0.053 \\
Crystal Density - $\rho_{o}$ & $1.9 \mathrm{~g}^{3} \mathrm{~cm}^{3}$ \\
Sound Speed $-c_{o}$ & $2.74 \times 10^{5} \mathrm{~cm} / \mathrm{s}$ \\
Slope of $U_{s}-U_{p}$ Hugoniot $-s$ & 2.6 \\
Gruneisen Parameter, $-\Gamma_{\mathrm{o}}$ & 1.1 \\
Specific Heat $-c_{v}$ & $1.45 \times 10^{11} \mathrm{erg} / \mathrm{gm}-\mathrm{ev}$ \\
Yield Stress $-Y$ & $1.0 \mathrm{Kbar}$ \\
Poisson Ratio $-v$ & 0.25 \\
Fracture Stress $-\sigma_{f}$ & $-20.0 \mathrm{Kbar}$ \\
\hline
\end{tabular}

A Mie-Grüneisen equation of state is used for the HMX and an elastic-perfectly plastic model, with yield at $1 \mathrm{Kbar}$, is included for material strength. A list of model parameters is given in Table 1.

In the first set of calculations, inert HMX "crystals" are impacted at $1000 \mathrm{~m} / \mathrm{s}$. Although it is recognized that the induced stress levels are sufficiently high to trigger reaction, the focus of the calculation centers on the thermal-mechanical behavior without the complication of chemical reaction. Thermal conduction effects are also included in these simulations.
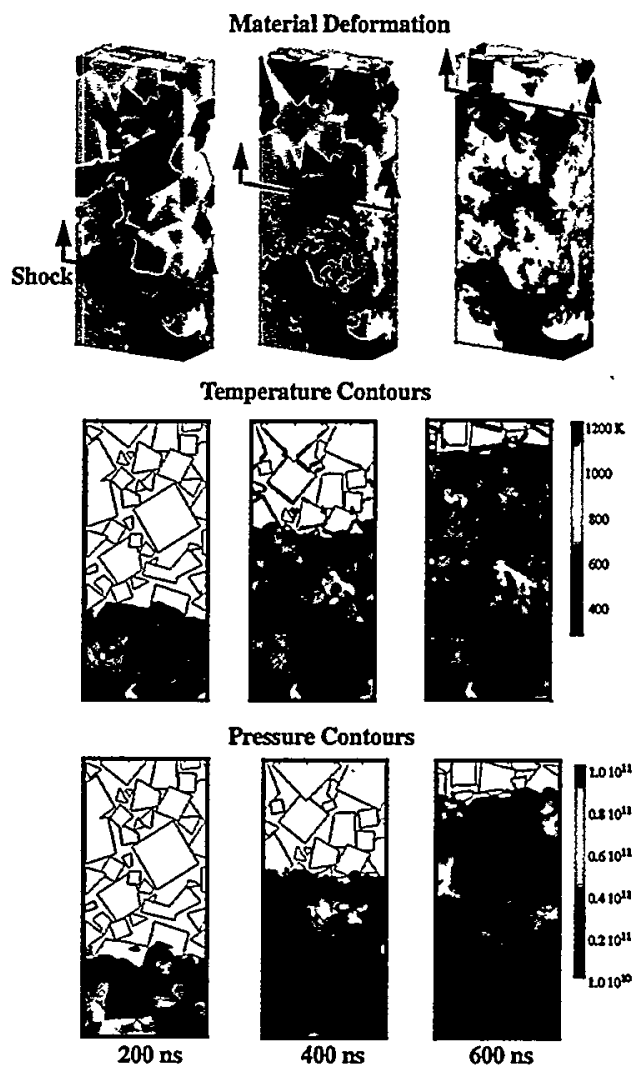

Fig. $11000 \mathrm{~m} / \mathrm{s}$ impact on HMX Holston 920-32. Upper time planes are materials, center are temperatures and lower are pressures.

Symmetric lateral boundary conditions are placed on the computational domain and at the impact boundary a hard wall condition is imposed. 
A reverse-ballistics calculation is represented by material stagnating at a hard wall boundary. This produces multiple shocks that pass back into the "crystal" array. The resolution of these calculations, using a uniform $5 \mu \mathrm{m}$ cell size, requires well over $\mathbf{2 7 . 5}$ million computational cells. Figure 1 displays a midplane cross-section of the material interfaces, pressure and temperature contours for three $200 \mathrm{~ns}$ intervals after impact. The simulations display the details of the rapid deformation occurring at material contact points. The nature of the dispersive fields includes large amplitude fluctuations of stress with wavelengths of several particle diameters. These fluctuating fields are due to the effects of shocks interacting with individual material surfaces and multiple crystal interactions. Interestingly, the amplitude of the fluctuating pressure field is of the same order as the mean pressure state.

Localization of energy produces "hot-spots" due to shock focusing and plastic work as material flows into interstitial regions. The effect of thermal dissipation leads to a decay of the "hot-spot" temperatures. It is the race between energy localization and dissipation that dictates the initiation and sustaining of reaction. Numerical simulations suggest that the "hot-spots" scale directly to particle size.

As a comparative numerical simulation, impact on plastic bonded material is modeled with the HMX "crystals" and polyurethane binder (shown in Figure 2). Here the interstitial regions are filled with the polymeric binder. As often seen in real materials, voids are included as spherical inclusions placed randomly in the mixture. In this example, a void volume fraction of $\sim 5 \%$ is incorporated in the binder material. The binder is represented by an appropriate tabular equation of state for polyurethane. An elastic-perfectly plastic material strength model is applied with a yield stress of $\sim 0.1 \mathrm{Kbar}$ and a Poisson's ratio of $\sim 0.45$. As in the prior calculation, all material interfaces are treated as frictionless. The material surfaces are displayed along with midplane contours of temperature and pressure at $100 \mathrm{~ns}$ intervals after impact.
In this example of a plastic-bonded energetic material, multiple shocks arise that move at nearly twice the velocity of that seen in the prior HMX simulation due to the higher density of material.

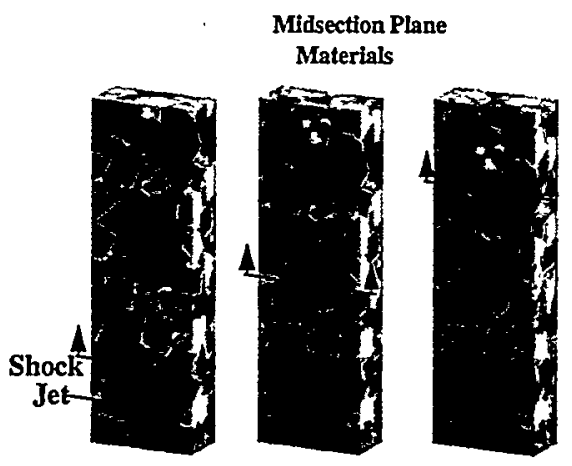

Temperature Contours

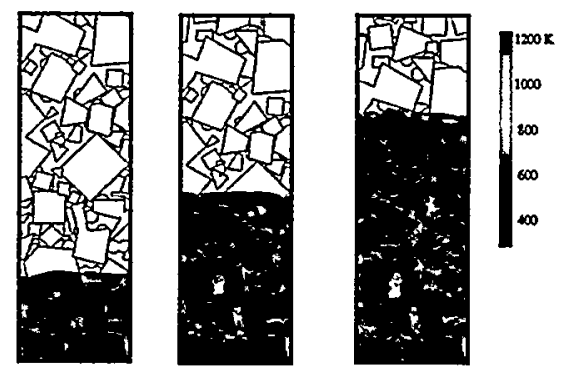

Pressure Contours
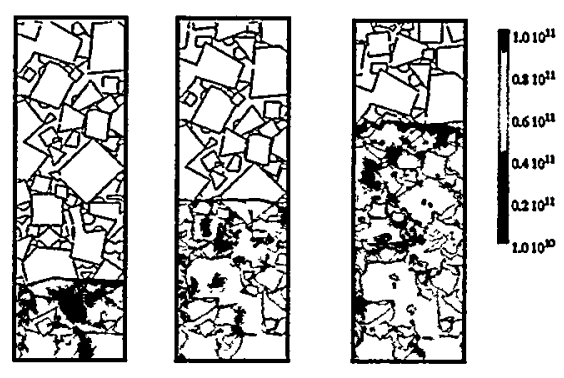

$300 \mathrm{~ns}$

Fig. $21000 \mathrm{~m} / \mathrm{s}$ impact on HMX Holston $920-32$ with polyurethane binder and $5 \%$ void volume.

Since the pores are filled with polyurethane and voids, the shocks pass through material interfaces having different shock impedance characteristics and multiple waves coalesce and release as the 
crystals interact with themselves and with the binder.

As the shocks interact with the voids, locallyaccelerated interfaces produce jets that penetrate into adjacent "crystals." As one would expect, much of the material distortion occurs in the binder material; however, the effect of shock focusing and jetting into the HMX crystals is evident. Again, the stress field exhibits fluctuations and nonequilibrium behavior and patterns of wave coalescence and rarefaction associated with the multiple waves having Mach stems and non-planar fronts.
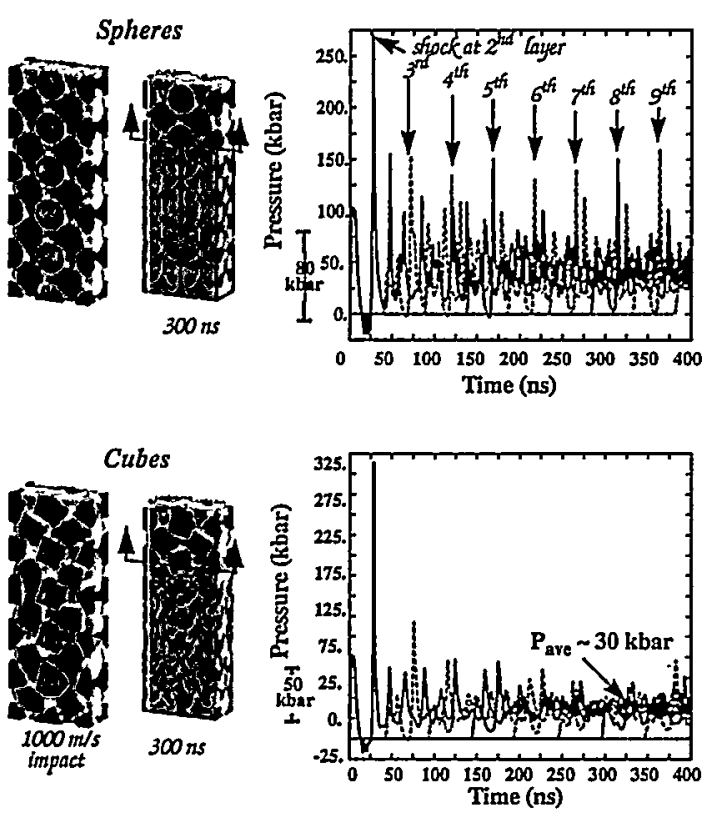

Fig. 3 Stress histories along the centerline of the nonreactive HMX crystal array modeled as ordered spherical particles vs. randomly-oriented cubes of size $100 \mu \mathrm{m}$.

To investigate the effect of crystal shape, simulations are conducted for a $1000 \mathrm{~m} / \mathrm{s}$ impact on an ordered array of single size $(100 \mu \mathrm{m}) \mathrm{HMX}$ approximated as spheres or cubes with random orientation. In contrast to smoothed particle surfaces, the effects of angular morphology are assessed. Lagrangian tracer points are included at the center of each HMX "crystal" along the centerline. At these locations, the stresses are monitored and an overlay of the centerline pressure histories are given in Figure 3. As seen in these simulations, fluctuating fields are due to the effects of shocks interacting with individual material surfaces and contact points.

In this calculation, the mean pressure field is $\sim 40 \mathrm{Kbar}$ and the amplitudes of the shocks traversing the "crystals" are of the same order. These highly fluctuating stress states persist over several particle diameters. The pressure histories of the ordered spherical particles exhibit higher pressure fluctuations than the cubic system due to resonance. As one might expect, disorder of particle orientation reduces the resonance effect.

\section{MESOSCALE REACTIVE MODEL}

In the previous section, numerical simulations considered only thermal and mechanical effects without the effect of reaction. At the $1000 \mathrm{~m} / \mathrm{s}$ impact condition the localized temperatures are seen to exceed those required for prompt reaction and an appropriate model for chemistry must include multi-step endothermic and exothermic mechanisms similar to those proposed by McGuire and Tarver (13) and kinetics rate data such as measured in diamond anvil studies by Russel, et al. (14). Multi-step chemistry with multi-state formulations, similar to those used in finite element analysis (15), will be implemented in shock physics codes in the near future.

An ignition/growth two-state history variable reactive burn (HVRB) model (16) is applied in this study. In this approach, reaction is triggered at a pressure threshold (as opposed to a temperature criteria) and a pressure-dependent rate law describes the extent of reaction. A single reaction progress variable has generally led to good agreement with experimental run-to-detonation Pop-plot and other data. Thus, the application of this approach assumes that all reaction pathways are self-similar to averaged state data. Utilization of this model approach provides insight as to what can be expected when more physically-based combustion 
descriptions are determined for the crystalline HMX.

In the following example, a numerical simulation is shown with a spatial extent that bridges the mesoscale to the macroscale. In this calculation about 1900 HMX crystals are impacted by a copper flyer plate at $1000 \mathrm{~m} / \mathrm{s}$ and reaction is triggered by the HVRB model. Reaction parameters are set for short run distance-to-detonation in order to explore the nature of the detonation wave through the random crystal structure.

Figure 4 displays a computational space defined by about 12 million evenly-spaced $5 \mu \mathrm{m}$ cells. The crystal array considers the HMX Holston 920-32 particle size distribution packed to a $68 \%$ TMD density. (A dime-sized sample of 190,000 crystals resolved with 1.3 billion cells required using the entire Sandia TFLOP parallel computer with 4500 processors and $104 \mathrm{MBs}$ /processor).

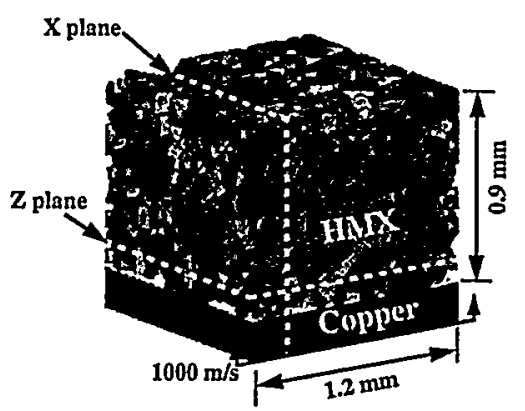

Fig. 4 HMX crystal array and $0.2 \mathrm{~mm}$ thick copper plate impacting at $1000 \mathrm{~m} / \mathrm{s}$.

To display the transient initiation and growth of reaction at the grain scale, it is convenient to display several cross-sectional cut planes of pressure contours at various times. Figure 5 displays the $x$ and $z$ planes at times 10,30 and $50 \mathrm{~ns}$ after initial contact with the copper impactor plate. It is noted that spatial fluctuations also occur in directions normal to these two-dimensional surfaces.

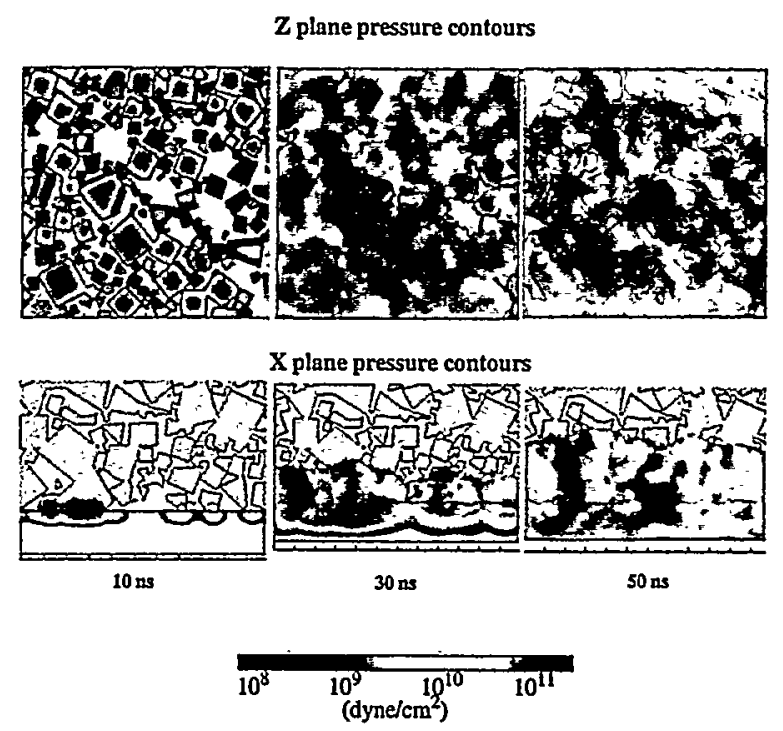

Fig. 5 Transient structure of mesoscale detonation wave growth and propagation in 68\% TMD HMX.

In the top time sequence of contour plots, initiation of rapid reaction is seen to first occur in the large "crystals". Lower sustained stresses occur in smaller particles due to rarefaction effects at grain surface boundaries. As the reactive wave proceeds from crystal-to-crystal, coalesced shocks produce Mach stems. These shocks interact with grain boundaries and as the reaction nears completion they merge and the wavelength of the localized stress field grows in spatial extent. In this simulation, localized pressures exceed $400 \mathrm{Kbar}$. Clearly, the detonation wave structure of reactive heterogeneous materials is far more complex and greatly different than that based on singular state analysis.

\section{SUMMARY AND CONCLUSIONS}

This study has focused on providing detailed wave information needed to define the appropriate length and time scales for new measurement techniques (17) that may lead to a better understanding of the processes associated with mechanical initiation of granular energetic materials. Continuum mixture modeling has been successfully applied to replicate impact measurements; however, these 
ensemble-averaged models are inadequate and do not currently include descriptions of the highly transient fields near shock fronts. These effects may be the key to describing shock initiation of heterogeneous materials. Work is in progress to apply averaging methods and data interrogation techniques to the extensive numerical data from mesoscale simulations to determine constitutive models that can then be used in continuum descriptions.

Mesoscale modeling has been applied to the study of dispersive waves experimentally observed in prior reported work. Detailed shock physics analyses reveal that the shocked heterogeneous materials exhibit highly fluctuating stress states and the localization of energy due to plastic deformation and crystal interactions. This modeling suggests that the shock waves (or even detonations) in heterogeneous materials are not single jump states. Future modeling is planned to investigate the effect of defects within crystals (such as voids or shear bands), blends of material (such as metals) and improved material strength, interface interactions and reaction models.

\section{ACKNOWLEDGEMENTS}

I would like to thank M. Kipp (SNL) for help with the computational work and F. van Swol (SNL) for assistance in the MC/MD modeling. The many fruitful discussions with R. A. Graham (SNL), S. Sheffeld (LANL), C. Tarver (LLNL) and C-O Leiber (WIWEB) are also gratefully acknowledged.

\section{REFERENCES}

1 Bowden, F. P. and Yoffe, A. D., Initiation and Growth of Explosions in Liquids and Solids, Cambridge University Press, Cambridge, 1952.

2 Van der Steen, A.C., Verbeek, H.J., and Meulenbrugge, J. J., "Influence of RDX Crystal Shape on the Shock Sensitivity of PBX's," Ninth Symposium (International) on Detonation, Portland, Oregon, pp. 83-88, 1989.

3 Bome, L., "Influence of Intragranular Cavities of RDX Particle Batches on the Sensitivity of Cast Wax Bonded Explosives," Tenth Symposium (International) on Detonation, Boston, Massachusetts, pp. 286-293, 1993.
4 Sheffield, S. A., Gustavsen, R. L., Alcon, R. R., Graham, R. A., and Anderson, M. U., "Particle Velocity and Stress Measurements in Low Density HMX," High-Pressure Science and Technology (Eds Schmidt, Shaner, Samara and Ross), pp. 1377-1380, 1994.

5 Coffey, C. S., "The Initiation of Explosive Crystals by Shock or Impact," Tenth Symposium (International) on Detonation, Boston, Massachusetts, pp. 824-830, 1993.

6 Drew, D. A. and Passman, S. L., Theory of Multicomponent Fluids, Applied Mathematics Series, Vol 135, Springer, 1998.

7 Baer, M. R., "Continuum Mixture Modeling of Reactive Media," High-Pressure Shock Compression of Solids, IV, Response of Highly Porous Solids to Shock Loading, Chapter 3, Springer-Verlag, 1996.

8 McGlaun, J. M., Thompson, S. L., and Elrick, M. G., "CTH: A Three-Dimensional Shock Wave Physics Code," Int. J. Impact Eng., Vol 10, 351-360, 1990.

9 Cesarano, J., McEuen, M., and Swiler, T.,"Computer Simulations of Particle Packing," SAND96-1703, Sandia National Laboratories, Albuquerque, NM, 1996.

10 van Swol, F., personal communication.

11 Tanemura, M., "Models and Simulations of Random Structure of Particles," Acta Stereol, pp 41-52, 1991.

12 Dick, J. J., "Measurements of the Shock Initiation Sensitivity of Low Density HMX," Combustion and Flame, Vol 54,pp 121-129, 1983.

13 McGuire, R. R., and Tarver, C. M., "Chemical Decomposition Models for the Thermal Explosion of Confined HMX, TATB, RDX, and TNT Explosives," Sixth Symposium (International) on Detonation, NSWC MP 82-334, p 56, 1981.

14 Russell, T. P., Allen, T. M., Gupta, Y. M., "Time Resolved Optical Spectroscopy to Examine Chemical Decomposition of Energetic Materials Under Static High Pressure and Pulsed Heating Conditions," Materials Research Society Proc., Vol 418, pp 385-390, 1996.

15 Baer, M. R., Gross, R. J., Gartling, D. K., and Hobbs, M. L., "Multidimensional Thermal-Chemical Cookoff Modeling," JANNAF Prop. Sys. Haz Sub. Mtg., San Diego, CA, 1994.

16 Kerley, G. I., "CTH Equation of State Package: Porosity and Reactive Burn Models," SAND92-0553, Sandia National Laboratories, 1992.

17 Trott, W.M., Knudson, M.D., Chhabildas, L.C., and Assay, J.R., "Measurements of Spatially-Resolved Velocity Variations in Shock Compressed Heterogeneous Materials Using a Line-Imaging Velocity Interferometer," (this volume). 